\title{
The U Sign: Tenth Landmark to the Central Region on Brain Surface Reformatted MR Imaging
}

M. Wagner, A. Jurcoane, and E. Hattingen

\begin{abstract}
BACKGROUND AND PURPOSE: Identification of the central region is of special importance to avoid neurologic deficits in brain surgery. Brain surface reformatted images (Mercator view) map the frontoparietal brain surface in 1 view and provide a synopsis of the most important landmarks. In this view, the U-shaped subcentral gyrus appears as a distinctive anatomic structure enclosing the Sylvian end of the central sulcus. The purpose of this study was to add the subcentral gyrus as a new landmark to the central region ( $U$ sign) and to compare its frequency and applicability with common landmarks in healthy hemispheres.
\end{abstract}

MATERIALS AND METHODS: Mercator views of 178 hemispheres in 100 patients were generated from 3D MR imaging datasets. The hemispheres were evaluated on Mercator views for the presence or absence of each of the 9 common landmarks and the new $U$ sign identifying the central region.

RESULTS: The new landmark U sign was most common (96.6\%), followed by the thin postcentral gyrus sign (95.5\%). The least common landmark was the $\Omega$-shaped handknob (54.5\%). None of the landmarks could be identified in all hemispheres. All landmarks could be identified bilaterally in only $1.3 \%$ of patients.

CONCLUSIONS: On the Mercator view, the new $U$ sign is an applicable and even the most frequent landmark to identify the central region. Considering the variability of the anatomic structures of the brain, including the motor hand area, the synopsis of all 10 landmarks on this surface-reformatting projection is a helpful adjunct to standard MR imaging projections to identify the central region.

ABBREVIATIONS: post cg = postcentral gyrus; pre cg = precentral gyrus; $s f g=$ superior frontal gyrus

D ue to the convexity of the surface of the brain, the identification of individual sulci and gyri can be difficult on axial MR imaging. The anatomic pattern of the surface of the brain may be further distorted by mass lesions or edema. However, reliable identification of the central region, including the primary somatomotoric and somatosensoric cortices, is crucial for diagnostic and therapeutic issues. Pathologies in close proximity to the central region should be identified accurately to avoid severe neurologic deficits from surgery. ${ }^{1-4}$

Advanced techniques like presurgical functional MR imaging and pre- and intraoperative electrophysiologic examinations are the most adequate methods of identifying and locating brain function. However, conventional MR imaging is primarily re-

Received March 2, 2012; accepted after revision May 4.

From the Institute of Neuroradiology, University Hospital, Goethe University Frankfurt, Frankfurt am Main, Germany.

Please address correspondence to Marlies Wagner, MD, Institute of Neuroradiology, Goethe University Hospital, Schleusenweg 2-16, D-60528 Frankfurt am Main, Germany; e-mail: marlies.wagner@kgu.de

http://dx.doi.org/10.3174/ajnr.A3205 quired to depict the morphology and topography of mass lesions in relation to brain anatomy.

A variety of methods and several landmarks have been described to identify the central region. ${ }^{1,2,5-12}$ Previously, sectional CT and MR imaging were evaluated for landmarks that were only visible on axial or sagittal image orientations. Therefore, this approach often required the time-consuming evaluation of more images with different orientations.

The Mercator view is a curved multiplanar reformatting method based on 3D MR imaging datasets, which can be processed on every MR imaging workstation in a few minutes. It shows the brain surface of both hemispheres from the upper convexity to the Sylvian fissure, and it facilitates the identification of the brain surface anatomy even in cases when this is disturbed due to mass lesions. ${ }^{413-15}$ It has been shown that brain surface reformatted imaging visualizes 9 of the most important landmarks in 1 view. ${ }^{4}$ Evaluating the Mercator view, we recognized the subcentral gyrus as a distinctive U-shaped anatomic structure. Therefore, our purpose was to introduce this $U$ sign as the tenth landmark that connects the precentral with the postcentral gyrus. The 




FIG 1. Example of the thin post cg sign, and the midline sulcus sign. The precentral gyrus has a thicker anteroposterior diameter llarge white arrow) compared with the postcentral gyrus (small white arrow). The longest sulcus running horizontally and entering the interhemispheric fissure (black arrow) is the central sulcus.

applicability and frequency of this new landmark were evaluated in healthy hemispheres on the Mercator view and were compared with the other 9 landmarks modified from Naidich and Brightbill. ${ }^{2}$

\section{MATERIALS AND METHODS}

One hundred consecutive patients ( 5 months to 84 years of age) underwent 3T MR imaging (Verio; Siemens, Erlangen, Germany) including a T1- or T2-weighted 3D sequence in a sagittal orientation. Data were transferred to the built-in workstation (syngo X Workplace; Siemens) for postprocessing by using the built-in software Numaris/4 (syngo MR B17; Siemens). Planar surface reformations ("pancake-view," Mercator projection) from an angled coronal view to the central sulcus (in the sagittal view) were generated in a manner similar to previously described procedures. ${ }^{13,14}$ Thus, both hemispheres were reformatted from the interhemispheric fissure medially to the Sylvian fissure laterally, resulting in 19 sections (2-mm thickness and 2-mm intersection gap; FOV, $250 \mathrm{~mm}$; rectangular FOV, $100 \% ; 179$ encoding steps; isotropic voxel, $1 \mathrm{~mm}^{3}$ ). The whole procedure takes no longer than 1 minute.

Only hemispheres without mass lesions were included for further analysis. Images were consensually evaluated by 2 experienced neuroradiologists (M.W., E.H.) describing the presence or absence of each of the 10 landmarks described hereafter.

Modified after Naidich and Brightbill, ${ }^{2} 10$ ways to identify the central region were defined as listed below (Figs 1-4):

1) The upper T sign: The intersection of the well-identifiable superior frontal sulcus with the precentral sulcus has the shape of an upper case $T$. The pre cg can be found immediately posterior to this T shape.

2) The L sign: The sfg terminates in the pre cg, which runs laterally from the posterior end of the sfg, therefore together forming an upper case $L$.

3) The lower $T$ sign: The inferior frontal sulcus posteriorly terminates in the pre sc, also in the shape of an upper case $T$ that is dorsally bordered by the pre cg.

4) The $M$ sign: The typical upper case $M$ shape of the inferior

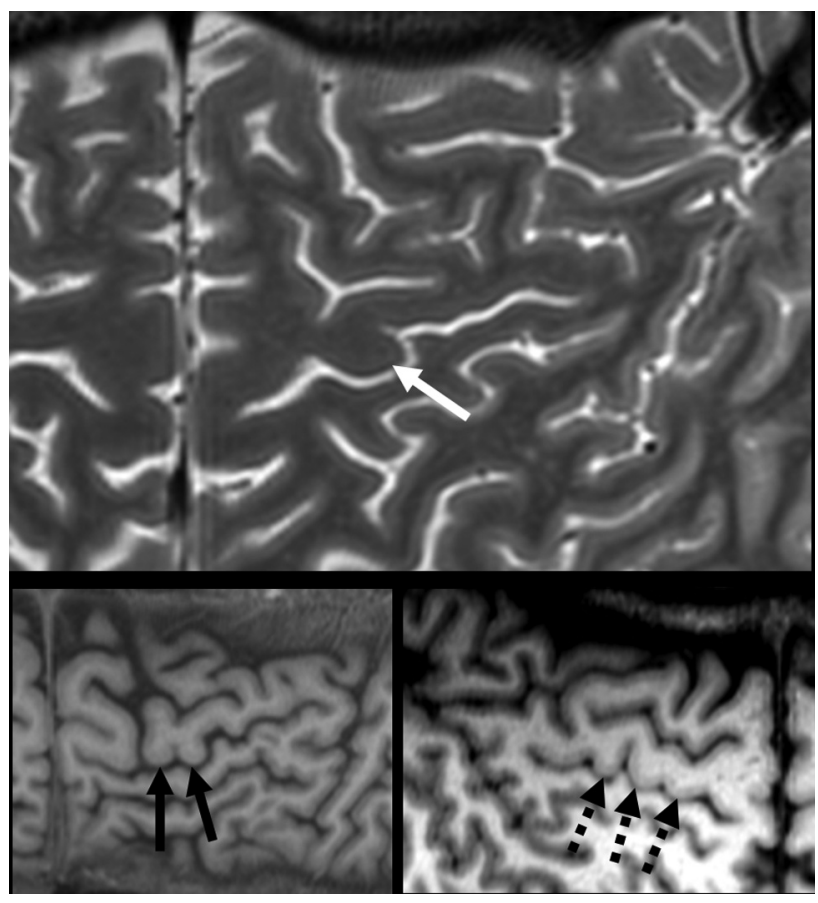

FIG 2. Variants of the handknob. In our study, we only considered the inverted $\Omega$ shape (white arrow) because other variants, such as inverted $\varepsilon$ (continuous black arrows), and multiple bulgings (dotted black arrows) were not assessed.

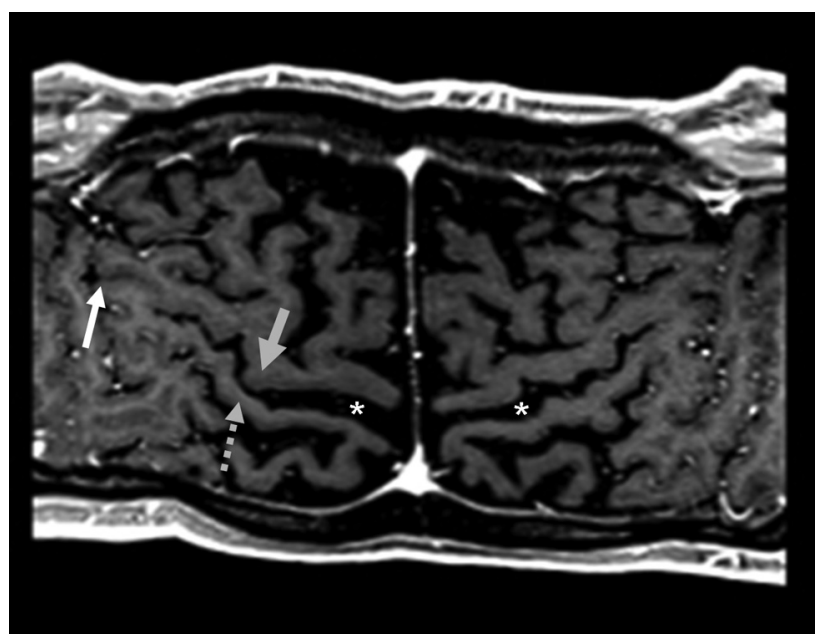

FIG 3. The $U$ sign. Right hemisphere. Absence of the subcentral gyrus as a rare variant, the central sulcus (asterisk) terminates into the Sylvian fissure (white arrow). Gray arrows mark the precentral (continuous arrow) and postcentral (dotted arrow) gyri. Left hemisphere: The subcentral gyrus connects the pre- and postcentral gyri (U sign).

frontal gyrus is formed by its partes orbitalis, triangularis, and opercularis. If one followed the $M$, it terminates medially into the pre cg.

5) The bifid post cg sign: Toward the interhemispheric fissure, the postcentral gyrus is medially fissured, enclosing the pars marginalis of the cingulated sulcus with both legs and thereby forming the characteristic bifid post cg sign.

6) The midline sulcus sign: The longest sulcus running horizontally and entering the interhemispheric fissure is the central sulcus (Fig 1).

7) The bracket sign: The bihemispheric symmetric pars marginalis of the cingulate sulcus forms an anteriorly opened bracket. 


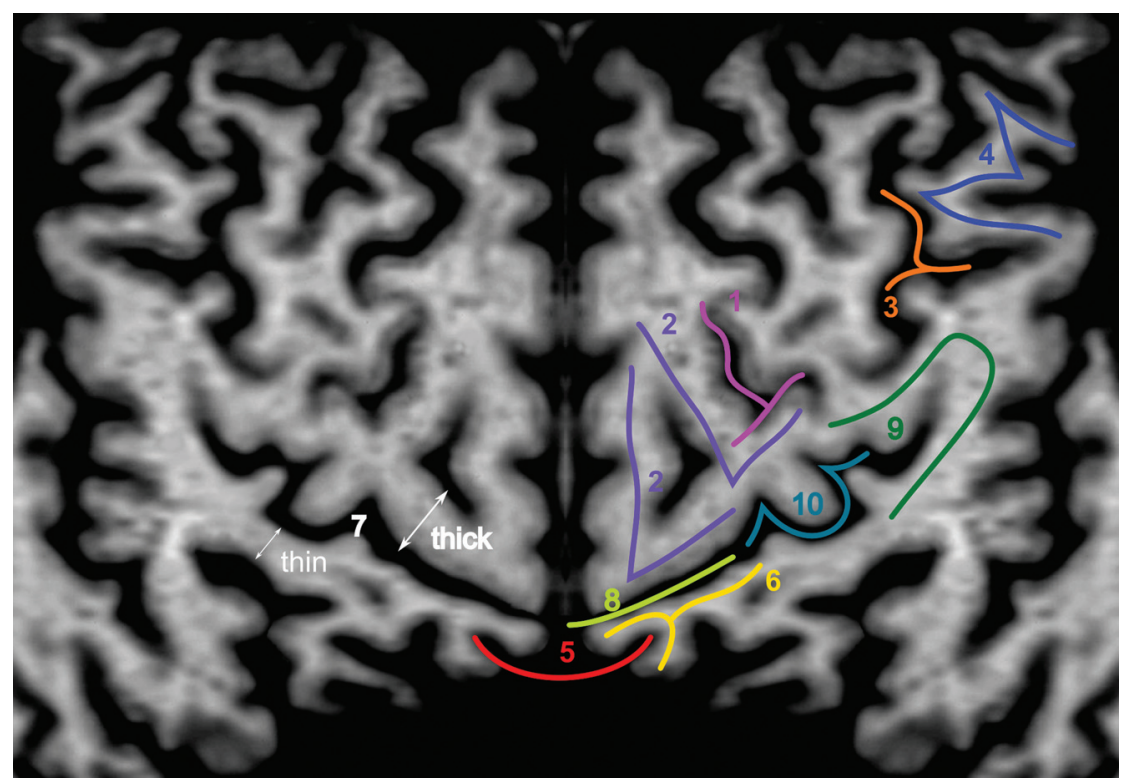

FIG 4. Examples of all 10 landmarks in 1 single section: 1 indicates the upper $T$ sign; 2 , the $L$ sign (duplicated as a variant); 3 , the lower T sign; 4, the M sign; 5 , the bracket sign; 6 , the bifid post cg sign; 7 , the thin post cg sign (fenestrated post cg as a variant); 8 , the midline sulcus sign; 9 , the subcentral gyrus sign; 10, the handknob. Only 1 hemisphere is depicted and was mirrored for better visualization.

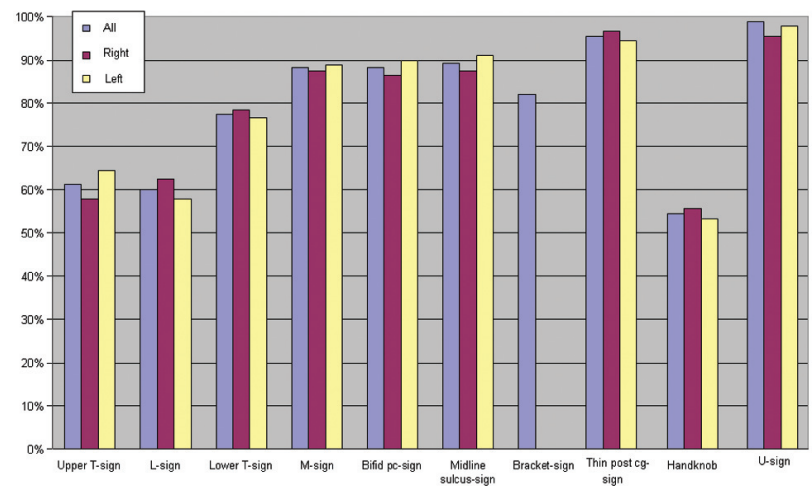

FIG 5. Barplots representing the frequency of landmark identification as a percentage of all (blue), right (purple), and left (yellow) hemispheres.

The rostrally adjacent gyrus is the post cg; the adjacent sulcus is the central sulcus.

8) The thin post cg sign: On the surface, the precentral gyrus has a thicker anteroposterior diameter compared with the postcentral gyrus, which is thinner (Fig 1).

9) The handknob: We defined the "handknob" as 1 single $\Omega$-shaped dorsally convex bulge of the precentral gyrus (Fig 2).

Furthermore, we introduced the U sign: The first continuous sulcus, which is enclosed by a perisylvian U-shaped gyrus, can be identified as the central sulcus (Fig 3).

\section{RESULTS}

One hundred patients ( 43 female) between 5 months and 84 years of age were investigated. From 100 patients, 178 hemispheres could be included in the analysis. The remaining 22 hemispheres had to be excluded due to surface-disturbing pathologies. In 78 patients, both hemispheres could be analyzed. Altogether, 88 of the included hemispheres were right (49.4\%) and 90 were left $(50.6 \%)$ hemispheres.

In only 1 of the 78 patients (1.3\%), all landmarks could be identified bilaterally. None of the 10 landmarks could be identified in all hemispheres. The most frequent landmarks were the subcentral gyrus (96.6\% in total, $95.5 \%$ right, $97.8 \%$ left) and the thin post cg $(95.5 \%$ in total, $96.6 \%$ right, $94.4 \%$ left). Least often identifiable was the $\Omega$-shaped handknob of the precentral gyrus as defined above $(54.5 \%$ in total, $55.7 \%$ right, 53.3\% left) (Fig 5).

See the Table for the frequency of all landmarks.

\section{DISCUSSION}

The $U$ sign defining the $U$ shape of the subcentral gyrus should be considered a new landmark to identify the central sulcus on the Mercator view. With this brain surface reformatting projection, the $U$ sign appears more frequently than 9 established landmarks previously defined on standard axial and sagittal sections. ${ }^{4,14}$ Therefore, the $\mathrm{U}$ sign is a very helpful addition to the synopsis of landmarks that can be found on the Mercator view to enhance the diagnostic accuracy in detecting the central region.

The subcentral gyrus is a U-shaped convolution that connects the precentral and postcentral gyri, marked by the anterior and posterior subcentral branch of the Sylvian fissure. On sectional MR imaging, the subcentral gyrus is best seen on sagittal images as the rolandic operculum, which encircles the central sulcus and thereby separates the central sulcus from the Sylvian fissure. ${ }^{5}$ On the Mercator view, the perisylvian frontal and rolandic opercula are well-depicted in the continuity of the upper convexity so that we could define the $\mathrm{U}$ sign as follows: The first continuous sulcus that is enclosed by a perisylvian $\mathrm{U}$-shaped gyrus can be identified as the central sulcus. The $\mathrm{U}$ sign was found in $96.6 \%$ of the healthy hemispheres. Two previously described variations ${ }^{1,11}$ were only seen once each in our collective of healthy hemispheres. One variant is the longitudinally fissured subcentral gyrus, resulting in a junction of the central fissure with the Sylvian fissure (5\%-19\%) (Fig 3). The other rare variant describes a second parenchymal bridge connecting the pre- and postcentral gyri medially to the subcentral gyrus.

In addition, the present study revealed that on mercator view, anatomic variations of the middle precentral gyrus become more evident and may be confusing. In this sense, one of the most popular and frequent landmarks on axial sections, the handknob, was less commonly identified on the Mercator view. The handknob represents the motor hand area and describes an $\Omega$-formed dorsal bulging of the precentral gyrus. ${ }^{12}$ The $\Omega$-formed or hooklike motor hand area was found in $90 \%-98 \%$ of axial sections. ${ }^{2,6,12}$ Some variability of the motor hand area was already described in axial images as an $\varepsilon$-formed shape ${ }^{12}$ and as the variant of multiple bulgings. However, the Mercator-like unfolding of the central region revealed a higher variability of the bulgings in 
Frequency of all landmarks

\begin{tabular}{lccc}
\hline & $\begin{array}{c}\text { All Hemispheres } \\
(\boldsymbol{n}=178)\end{array}$ & $\begin{array}{c}\text { Right } \\
(\boldsymbol{n}=\mathbf{8 8})\end{array}$ & $\begin{array}{c}\text { Left } \\
(\boldsymbol{n}=\mathbf{9 0})\end{array}$ \\
\hline Upper T sign & $109(61.2 \%)$ & $51(58 \%)$ & $58(64.4 \%)$ \\
L sign & $107(60.1 \%)$ & $55(62.5 \%)$ & $52(57.8 \%)$ \\
Lower T sign & $138(77.5 \%)$ & $69(78.4 \%)$ & $69(76.7 \%)$ \\
M sign & $157(88.2 \%)$ & $77(87.5 \%)$ & $80(88.9 \%)$ \\
Bifid pc sign & $157(88.2 \%)$ & $76(86.4 \%)$ & $81(90 \%)$ \\
Midline sulcus sign & $159(87.5 \%)$ & $77(87.5 \%)$ & $82(91.1 \%)$ \\
Bracket sign & 82\% (of 78 patients) & & \\
Thin post cg sign & $170(95.5 \%)$ & $85(96.6 \%)$ & $85(94.4 \%)$ \\
Handknob sign & $97(54.5 \%)$ & $49(55.7 \%)$ & $48(53.3 \%)$ \\
Subcentral gyrus sign & $176(98.9 \%)$ & $84(95.5 \%)$ & $88(97.8 \%)$ \\
\hline Note:-Bifid pc sign indicates the bifid postcg sign. & &
\end{tabular}

the middle of the precentral gyrus, which may be less obvious by sectioning the hemisphere in axial sections.

All other landmarks were identified with similar frequencies as previously described on axial or sagittal sections, ${ }^{1,2,5,6}$ with an occurrence ranging between $60.1 \%$ and $89.3 \%$. The thin post $\mathrm{cg}$ sign, ${ }^{1,2}$ describing the thinner anteroposterior diameter of the postcentral gyrus compared with the precentral gyrus, was detectable in $95.5 \%$. Naidich and Brightbill ${ }^{2}$ described the thin post cg sign as the most frequent landmark visible in $98 \%$ of axial MR imaging. This difference might be explained by the incomplete myelination of the central region during infancy, ${ }^{16,17}$ because all subjects in our study lacking this landmark by an equal thickness of the pre- and postcentral gyri were infants. The study collective of Naidich and Brightbill, ${ }^{2}$ however, included only children not younger than 4 years of age.

Only $1.3 \%$ of our healthy patients showed all 10 landmarks bilaterally. This intra- and interindividual variance of the cerebral surface anatomy ${ }^{1,18,19}$ may complicate the identification of the central sulcus on sectional slices. Therefore, the synopsis of different landmarks, including the new $\mathrm{U}$ sign on the Mercator view, should be used as an adjunct to standard MR imaging to improve diagnostic accuracy. This view continuously depicts the entire frontoparietal sulci and gyri without any sectioning, by unfolding the convex hemispheres to a flat map via multiplanar reformatting. However, the unfolding of the convex hemisphere leads to distortions, ${ }^{20}$ which increase toward the margins and deform the shapes and sizes of anatomic structures. This phenomenon is well-known from world maps like the Mercator projection, which gives a disproportionate image of areas, depending on their location from the equator. Furthermore, the Mercator view only depicts surface structures, so that sectional images are still needed to delineate the extension of a lesion into the deep of the brain. Therefore, the Mercator view cannot replace standard projections.

Nevertheless, it could be shown that the Mercator view is a simple and fast reformatting method to identify the central region, even in the presence of surface-disturbing mass lesions. ${ }^{4,14}$ However, we excluded hemispheres with pathologies to avoid confusing results.

\section{CONCLUSIONS}

The $\mathrm{U}$ sign is an applicable landmark to identify the central region on the brain surface reformatting projection Mercator view. This sign defines the $U$ shape of the subcentral gyrus, which merges the pre- and postcentral gyrus at the Sylvian fissure. Together with the synopsis of other landmarks depicted on the Mercator view, it may enhance diagnostic accuracy in identifying the central region. In this sense, the Mercator view should be an adjunct to standard MR imaging, keeping in mind that this unfolded map also leads to distortions of the true anatomic proportions.

\section{REFERENCES}

1. Naidich TP, Valavanis AG, Kubik S. Anatomic relationships along the low-middle convexity. Part I. Normal specimens and magnetic resonance imaging. Neurosurgery 1995;36:517-32

2. Naidich TP, Brightbill TC. Systems for localizing fronto-parietal gyri and sulci on axial CT and MRI. Int J Neuroradiol 1996;2:313-38

3. Tsuchiya K, Katase S, Hachiya J, et al. A new technique of surface anatomy MR scanning of the brain: its application to scalp incision planning. AJNR Am J Neuroradiol 1999;20:515-18

4. Hattingen E, Good C, Weidauer S, et al. Brain surface reformatted images for fast and easy localization of perirolandic lesions. J Neurosurg 2005;102:302-10

5. Naidich TP, Brightbill TC. The pars marginalis. Part 1: A bracket sign for the central sulcus in axial plane CT and MRI. Int J Neuroradiol 1996;2:3-19

6. Ebeling U, Huber P, Reulen HJ. Localization of the precentral gyrus in the computed tomogram and its clinical application. J Neurol 1986;233:73-76

7. Meyer JR, Roychowdhury S, Russell EJ, et al. Location of the central sulcus via cortical thickness of the precentral and postcentral gyri on MR. AJNR Am J Neuroradiol 1996;17:1699-706

8. Kido DK, Le May M, Levinson AW, et al. Computed tomographic localization of the precentral gyrus. Radiology 1980;135:373-77

9. Iwasaki S, Nakagawa H, Fukusumi A, et al. Identification of pre- and postcentral gyri on CT and MR images on the basis of the medullary pattern of cerebral white matter. Radiology 1991;179:207-13

10. Sobel DF, Gallen CC, Schwartz BJ, et al. Locating the central sulcus: comparison of MR anatomic and magnetoencephalographic functional methods. AJNR Am J Neuroradiol 1993;14:915-25

11. Ono M, Kubik S, Abernathey CD. Atlas of the Cerebral Sulci. Stuttgart, Germany: Georg Thieme Verlag; 1990

12. Yousry TA, Schmid UD, Alkadhi H, et al. Localization of the motor hand area to a knob on the precentral gyrus: a new landmark. Brain 1997;120:141-57

13. Bastos AC, Corneau RM, Andermann F, et al. Diagnosis of subtle focal dysplastic lesions: curvilinear reformatting from three-dimensional magnetic resonance imaging. Ann Neurol 1999;46:88-94

14. Hattingen E, Hattingen J, Clusmann $\mathrm{H}$, et al. Planar brain surface reformations for localization of cortical brain lesions. Zentralbl Neurochir 2004;65:75-80

15. Van Essen DC, Drury HA. Structural and functional analyses of human cerebral cortex using a surface-based atlas. J Neurosci 1997; 17:7079-102

16. Lebel C, Gee M, Camicioli R, et al. Diffusion tensor imaging of white matter tract evolution over the lifespan. Neuroimage 2012;60: $340-52$

17. Olesen PJ, Nagy Z, Westerberg H, et al. Combined analysis of DTI and fMRI data reveals a joint maturation of white and grey matter in a fronto-parietal network. Brain Res Cogn Brain Res 2003; $18: 48-57$

18. Steinmetz H, Fürst G, Freund HJ. Cerebral cortical localization: application and validation of the proportional grid system in MR imaging. J Comput Assist Tomogr 1998;13:10-19

19. Steinmetz H, Fürst G, Freund HJ. Variation of perisylvian and calcarine anatomic landmarks within stereotaxic proportional coordinates. AJNR Am J Neuroradiol 1990;11:1123-30

20. Carman GJ, Drury HA, Van Essen DC. Computational methods for reconstructing and unfolding the cerebral cortex. Cereb Cortex $1995 ; 5: 506-17$ 